\title{
REMARKS ON FREE PRODUCTS WITH RESPECT TO NON-TRACIAL STATES
}

\author{
YOSHIMICHI UEDA
}

\section{Introduction}

Since the appearance of D. Voiculescu's free probability theory detailed study on free products of von Neumann algebras (based on his theory) has been carried by several authors, and free group factors are their typical examples. Indeed, his theory gives us very powerful tools to analyze free group factors.

On the other hand, at the beginning of the 80's (i.e., before the appearance of D. Voiculescu's theory) S. Popa studied in detail free group factors and related type $\mathrm{II}_{1}$ factors and got several deep results ([P1], [P2]). He used there some techniques for type $\mathrm{II}_{1}$ factors related to his notion "orthogonal pairs". Then, at the mid 90's L. Ge generalized them to more general (but type $\mathrm{II}_{1}$ ) setting and solved some problems on maximal injective subalgebras ([G]).

In this note, we will try to generalize the techniques to more general and not necessary type $\mathrm{II}_{1}$ setting. These generalizations are rather natural, but enable us to obtain some results on free products with respect to non-tracial states. In fact, as applications a factoriality and type classification result for free products and a result on (normal) conditional expectations from free products onto their subalgebras will be obtained. Based on the latter result we will discuss the recent question posed by M. Izumi, R. Longo and S. Popa ([ILP]): Let $M \supseteq L \supseteq N$ be von Neumann algebras with a normal conditional expectation from $M$ onto $N$. If $N^{\prime} \cap M=\mathrm{C} 1$, does there exist a normal conditional expectation from $M$ onto $L$ ?

The author thanks H. Kosaki for discussions and Y. Watatani for his encouragement. He is also indebted to $\mathrm{M}$. Izumi for fruitful conversations. Finally, he thanks E. Størmer for useful comments making this paper more readable than the original version.

Received April 21, 1998; in revised form November 20, 1998. 


\section{Preliminaries}

Let $N_{i}, i=1,2$, be a $\sigma$-finite von Neumann algebra with a faithful normal state $\varphi_{i}$ respectively. The free product

$$
(M, \varphi)=\left(N_{1}, \varphi_{1}\right) *\left(N_{2}, \varphi_{2}\right)
$$

is a pair of a von Neumann algebra $M$ and a faithful normal state $\varphi$ characterized by the conditions ([V1], [VDN]):

(1) $M$ is generated by $N_{1}$ and $N_{2}$;

(2) $\left.\varphi\right|_{N_{i}}=\varphi_{i}, \quad i=1,2$;

(3) $N_{1}, N_{2}$ are free with respect to $\varphi$, i.e.,

$$
\varphi\left(x_{1}^{\circ} \cdots x_{n}^{\circ}\right)=0
$$

whenever $x_{j}^{\circ} \in N_{i_{j}}^{\circ}:=\operatorname{Ker} \varphi_{i_{j}}, i_{1} \neq \cdots \neq i_{n}$.

It is known that the modular automorphism $\sigma_{t}^{\varphi}$ satisfies

$$
\left.\sigma_{t}^{\varphi}\right|_{N_{i}}=\sigma_{t}^{\varphi_{i}}, \quad i=1,2 .
$$

(See [Br], [D1], [V1], [VDN].) Therefore, there exists a $\varphi$-conditional expectations $E_{i}: M \rightarrow N_{i}, i=1,2$.

\section{Some Properties of Free Products}

In this section some properties of free products (not necessary in the type $\mathrm{II}_{1}$ setting) will be shown.

Let $N_{1}, N_{2}, M, \varphi_{1}, \varphi_{2}, \varphi, E_{1}$ and $E_{2}$ be as in $\S 2$. Moreover, in what follows, $N_{i} \neq \mathrm{C} 1, i=1,2$ will be assumed.

Proposition 1. If $A$ is a non-atomic von Neumann subalgebra of the centralizer $\left(N_{1}\right)_{\varphi_{1}}$ and $x$ is an element in $M$ such that $x A x^{*} \subseteq N_{1}$, then $x$ is in $N_{1}$.

Proof. We make use of the idea in the proof of 2.5. Lemma of [P1] with some modifications.

Let $y^{\circ}$ be an alternating word in $N_{1}^{\circ}, N_{2}^{\circ}$ beginning and ending in $N_{2}^{\circ}, a, b$ be elements in $N_{1}$ with possibly $a=1$ or $b=1$, and $\left\{e_{i}\right\}_{i=1}^{n}$ be a partition of the unity of projections in $A$. We estimate 
REMARKS ON FREE PRODUCTS WITH RESPECT TO NON-TRACIAL STATES 113

$$
\begin{aligned}
\left|\varphi\left(x^{*}\left(a y^{\circ} b\right)\right)\right|^{2} & =\left|\varphi\left(\left(\sum_{i} e_{i}\right) x^{*}\left(a y^{\circ} b\right)\right)\right|^{2} \\
& =\left|\varphi\left(\sum_{i} e_{i} x^{*} a y^{\circ} b e_{i}\right)\right|^{2} \quad\left(e_{i} \in A \subseteq\left(N_{1}\right)_{\varphi_{1}} \subseteq M_{\varphi} \text { thanks to }(1)\right) \\
& \leq\left\|\sum_{i} e_{i} x^{*} a y^{\circ} b e_{i}\right\|_{\varphi}^{2} \quad(\text { Cauchy-Schwartz }) \\
& =\sum_{i}\left\|e_{i} x^{*} a y^{\circ} b e_{i}\right\|_{\varphi}^{2} \quad\left(\text { use } e_{i} e_{j}=0, i \neq j\right) \\
& =\sum_{i} \varphi\left(\left(e_{i} b^{*}\right) y^{\circ *}\left(a^{*} x e_{i} x^{*} a\right) y^{\circ}\left(b e_{i}\right)\right) \\
& =\sum_{i} \varphi_{1}\left(a^{*} x e_{i} x^{*} a\right) \varphi\left(e_{i} b^{*} y^{\circ *} y^{\circ} b e_{i}\right)
\end{aligned}
$$

(by $x e_{i} x^{*} \in N_{1}$ and the usual computation based on freeness)

$$
\begin{aligned}
& \leq\left\|y^{\circ}\right\|^{2}\|b\|^{2} \max _{i} \varphi_{1}\left(e_{i}\right) \varphi_{1}\left(a^{*} x\left(\sum_{i} e_{i}\right) x^{*} a\right) \\
& =\left\|y^{\circ}\right\|^{2}\|b\|^{2} \max _{i} \varphi_{1}\left(e_{i}\right) \varphi_{1}\left(a^{*} x x^{*} a\right) \\
& \leq\left(\|a\|\left\|y^{\circ}\right\|\|b|\|||x|)^{2} \max _{i} \varphi_{1}\left(e_{i}\right) .\right.
\end{aligned}
$$

Since $A$ is non-atomic, for each $\varepsilon>0$ we can choose $\left\{e_{i}\right\}_{i=1}^{n}$ in such a way that

$$
\varphi_{1}\left(e_{i}\right)<\frac{\varepsilon^{2}}{\left(\left\|a|||| y^{\circ}|\|||b||||x| \mid\right)^{2}\right.},
$$

and then the above estimate shows $\left|\varphi\left(x^{*}\left(a y^{\circ} b\right)\right)\right|<\varepsilon$. Therefore, $x$ is orthogonal to the word $a y^{\circ} b$.

Notice that the $*$-algebra $M_{0}$ (algebraically) generated by $N_{1}, N_{2}$ is

$$
M_{0}=N_{1}\left(=\mathrm{C} 1+N_{1}^{\circ}\right)+\operatorname{span}\left(\Lambda^{\circ}\left(N_{1}^{\circ}, N_{2}^{\circ}\right) \backslash N_{1}^{\circ}\right),
$$

and then

$$
L^{2}(M, \varphi)=L^{2}\left(N_{1}\right) \oplus \overline{\operatorname{span}\left(\Lambda^{\circ}\left(N_{1}^{\circ}, N_{2}^{\circ}\right) \backslash N_{1}^{\circ}\right)}\|\cdot\|_{\varphi} .
$$

Here $\Lambda^{\circ}\left(N_{1}^{\circ}, N_{2}^{\circ}\right)$ is the set of alternating words in $N_{1}^{\circ}, N_{2}^{\circ}$. The previous computation means that $x$ is orthogonal to every element in $\Lambda^{\circ}\left(N_{1}^{\circ}, N_{2}^{\circ}\right) \backslash N_{1}^{\circ}$, and hence $\left(1-e_{N_{1}}\right) x \xi_{\varphi}=0$, where $\xi_{\varphi}$ is an implementing vector for $\varphi$ and $e_{N_{1}}$ is the Jones projection associated with $E_{1}$. Therefore, $x \xi_{\varphi}=e_{N_{1}}\left(x \xi_{\varphi}\right)=E_{1}(x) \xi_{\varphi}$, and hence $x=E_{1}(x) \in N_{1}$.

Corollary 2. If $A$ is a non-atomic von Neumann subalgebra of the centralizer $\left(N_{1}\right)_{\varphi_{1}}$, then $A^{\prime} \cap M \subseteq N_{1}$. 
Proof. Let $u$ be an arbitrary unitary in $A^{\prime} \cap M$, and then $u A u^{*}=A \subseteq N_{1}$. Hence, by the previous proposition it is in $N_{1}$.

More generally, by the same reason as above we have the following corollary:

Corollary 3. If $A$ is a non-atomic von Neumann subalgebra of the centralizer $\left(N_{1}\right)_{\varphi 1}$, then $\mathscr{N}_{M}(A)=\mathscr{N}_{N_{1}}(A)$, where $\mathscr{N}_{M}(A)$ and $\mathscr{N}_{N_{1}}(A)$ mean the normalizers of $A$ in $M$ and $N_{1}$ respectively.

In his article ([R]) F. Rădulescu showed that the free product

$$
\left(L(\mathbf{Z}), \tau_{\mathbf{Z}}\right) *\left(M_{2}(\mathbf{C}), \varphi_{\lambda}\right)
$$

is a factor of type $\mathrm{III}_{\lambda}$. Here $\varphi_{\lambda}$ is the state associated with the density matrix $\operatorname{diag}\left(\frac{1}{1+\lambda}, \frac{\lambda}{1+\lambda}\right)$. The above corollary implies that (the canonical image of) $L(Z)$ is a singular MASA in the free product.

Set

$$
\tilde{M}:=M \rtimes_{\sigma^{\varphi}} \mathrm{R} \supseteq \tilde{N}_{i}:=N_{i} \rtimes_{\sigma^{\varphi_{i}}} \mathrm{R} \quad(i=1,2) .
$$

There exists a faithful normal conditional expectation $\hat{E}_{i}: \tilde{M} \rightarrow \tilde{N}_{i}$ such that

$$
\hat{E}_{i}\left(\int_{-\infty}^{\infty} x(t) \lambda(t) d t\right)=\int_{-\infty}^{\infty} E_{i}(x(t)) \lambda(t) d t
$$

for a smooth element $\int_{-\infty}^{\infty} x(t) \lambda(t) d t \in \tilde{M}$, where $E_{i}$ is as in $\S 2$. (See [KL] for example.) Note that it is the restriction of $E_{i} \otimes$ Id to $\tilde{M}$.

Corollary 4. If $A$ is a non-atomic von Neumann subalgebra of the centralizer $\left(N_{1}\right)_{\varphi_{1}}$, then $A^{\prime} \cap \tilde{M} \subseteq \tilde{N}_{1}$.

Proof. Since $\tilde{M}$ is contained in $M \otimes B\left(L^{2}(\mathrm{R})\right)$, we have

$$
A^{\prime} \cap \tilde{M} \subseteq\left(A^{\prime} \cap M\right) \otimes B\left(L^{2}(\mathrm{R})\right) \subseteq N_{1} \otimes B\left(L^{2}(\mathrm{R})\right)
$$

thanks to Corollary 2. Let $x$ be in $A^{\prime} \cap \tilde{M}(\subseteq \tilde{M})$. Now approximate $x$ by elements of the form $\int_{-\infty}^{\infty} x(t) \lambda(t) d t$ in the $\sigma$-weak topology. Then we have

$$
x=E_{1} \otimes \operatorname{Id}(x)=\lim \hat{E}_{1}\left(\int_{-\infty}^{\infty} x(t) \lambda(t) d t\right)=\lim \int_{-\infty}^{\infty} E_{1}(x(t)) \lambda(t) d t \in \tilde{N}_{1} .
$$

Here the first equality comes from (2) and the second comes from the fact that $\hat{E}_{1}$ is the restriction of $E_{1} \otimes \operatorname{Id}$ to $\tilde{M}$. 
Remark 5. By the similar method as in the proof of Proposition 1 we can also prove the following generalization of Theorem 4.1 of [P3]: Let $Q$ be a $\sigma$ finite von Neumann algebra with a faithful normal state $\psi$ and $N \supseteq L$ be $\sigma$ finite von Neumann algebras with a faithful normal conditional expectation $F$. Let

$$
(M, E)=(Q \otimes L, \psi \otimes \mathrm{Id}) *_{L}(N, F)
$$

be the amalgamated free product (see [P3], [U2], [VDN] for definition). If $A$ is a non-atomic von Neumann subalgebra of the centralizer $Q_{\psi}$, then

$$
(A \otimes \mathrm{C} 1)^{\prime} \cap M=\left(A^{\prime} \cap Q\right) \otimes L .
$$

The following proposition, which is a generalization of the result of L. Ge ([G: Lemma 5.1]), is worth pointing out:

Proposition 6. If $A$ is a non-atomic von Neumann subalgebra of the centralizer $\left(N_{1}\right)_{\varphi_{1}}$ and $x$ is an element in $M$ such that $x A x^{*} \subseteq N_{2}$, then $x=0$.

Proof. Let $\Lambda^{\circ}\left(N_{1}^{\circ}, N_{2}^{\circ}\right)$ be as in the proof of Proposition 1, and the $*$-algebra

$$
M_{0}=N_{2}\left(=\mathrm{C} 1+N_{2}^{\circ}\right)+\operatorname{span}\left(\Lambda^{\circ}\left(N_{1}^{\circ}, N_{2}^{\circ}\right) \backslash N_{2}^{\circ}\right)
$$

is $\sigma$-weakly dense in $M$.

Let $\left\{e_{i}\right\}_{i=1}^{n}$ be a partition of the unity of projections in $A$. For an alternating word $y^{\circ}$ in $N_{1}^{\circ}, N_{2}^{\circ}$ beginning and ending in $N_{1}^{\circ}$ and $a, b \in N_{2}$ with possibly $a=1$ or $b=1$, we estimate, by the similar way as in the proof of Proposition 1,

$$
\begin{aligned}
\left|\varphi\left(x^{*}\left(a y^{\circ} b\right)\right)\right|^{2} & =\left|\varphi\left(\sum_{i} e_{i} x^{*} a y^{\circ} b e_{i}\right)\right|^{2} \\
& \leq \sum_{i}\left\|e_{i} x^{*} a y^{\circ} b e_{i}\right\|_{\varphi}^{2} \\
& =\sum_{i} \varphi\left(e_{i} b^{*} y^{\circ *}\left(a^{*} x e_{i} x^{*} a\right) y^{\circ} b e_{i}\right) \\
& =\sum_{i} \varphi_{2}\left(a^{*} x e_{i} x^{*} a\right) \varphi\left(e_{i} b^{*} y^{\circ *} y^{\circ} b e_{i}\right)
\end{aligned}
$$

(by $x e_{i} x^{*} \in N_{2}$ and the usual computation based on freeness)

$$
\begin{aligned}
& \leq\left\|y^{\circ}\right\|^{2}\|b\|^{2} \max _{i} \varphi_{1}\left(e_{i}\right) \varphi_{2}\left(a^{*} x\left(\sum_{i} e_{i}\right) x^{*} a\right) \\
& \leq\left(\left\|a \left|\left\|y^{\circ}\right\|\|b|\|||x| \mid)^{2} \max _{i} \varphi_{1}\left(e_{i}\right) .\right.\right.\right.
\end{aligned}
$$

Thus, by the same way as in the proof of Proposition 1 we see that $x$ is or- 
thogonal to the word $a y^{\circ} b$, and hence to every word in $\Lambda^{\circ}\left(N_{1}^{\circ}, N_{2}^{\circ}\right) \backslash N_{2}^{\circ}$. Next, for $z \in N_{2}(\ni 1)$ we estimate

$$
\begin{aligned}
\left|\varphi\left(x^{*} z\right)\right|^{2} & \leq \sum_{i} \varphi\left(e_{i} z^{*} x e_{i} x^{*} z e_{i}\right) \\
& =\sum_{i} \varphi\left(e_{i}\left(z^{*} x e_{i} x^{*} z\right)\right) \quad\left(e_{i} \in A \subseteq\left(N_{1}\right)_{\varphi_{1}} \subseteq M_{\varphi} \text { thanks to }(1)\right) \\
& =\sum_{i} \varphi_{1}\left(e_{i}\right) \varphi\left(z^{*} x e_{i} x^{*} z\right) \quad(\text { by the same reason as above }) \\
& \leq \max _{i} \varphi_{1}\left(e_{i}\right) \varphi\left(z^{*} x\left(\sum_{i} e_{i}\right) x^{*} z\right) \\
& \leq\left(\|z|\|||x|)^{2} \max _{i} \varphi_{1}\left(e_{i}\right) .\right.
\end{aligned}
$$

Hence, $x$ is orthogonal to $z$.

Therefore, $x$ is orthogonal to $M_{0}$, and hence $x=0$.

\section{Factoriality and type classification for free products}

Let $N_{i}, i=1,2$, be a $\sigma$-finite von Neumann algebra with a faithful normal state $\varphi_{i}$, and

$$
(M, \varphi)=\left(N_{1}, \varphi_{1}\right) *\left(N_{2}, \varphi_{2}\right)
$$

be the free product. Assume that $N_{i} \neq \mathrm{C} 1, i=1,2$.

Set

$$
\tilde{M}:=M \rtimes_{\sigma^{\varphi}} \mathrm{R} \supseteq \tilde{N}_{i}:=N_{i} \rtimes_{\sigma^{\varphi_{i}}} \mathrm{R} \supseteq D:=\mathrm{C} \rtimes \mathrm{R} .
$$

There exists a faithful normal conditional expectation $E: \tilde{M} \rightarrow D$, which is the restriction of the Fubini map $\varphi \otimes$ Id to $\tilde{M}$. In [U2], it was shown that

$$
(\tilde{M}, E)=\left(\tilde{N}_{1},\left.E\right|_{\tilde{N}_{1}}\right) *_{D}\left(\tilde{N}_{2},\left.E\right|_{\tilde{N}_{2}}\right) .
$$

THEOREM 7. If either $\left(N_{1}\right)_{\varphi_{1}}$ or $\left(N_{2}\right)_{\varphi_{2}}$ contains a non-atomic von Neumann subalgebra, then $M$ is a factor and

$$
\mathscr{Z}(\tilde{M})=(\mathrm{C} \rtimes \mathrm{R}) \cap \mathscr{L}\left(\tilde{N}_{1}\right) \cap \mathscr{Z}\left(\tilde{N}_{2}\right),
$$

where $\tilde{M}, \tilde{N}_{1}, \tilde{N}_{2}$ are as in $\S 2$.

Proof. By symmetry we may and do assume that $\left(N_{1}\right)_{\varphi_{1}}$ contains a nonatomic von Neumann subalgebra. Since $N_{2} \neq \mathrm{C} 1$, we can choose orthogonal nonzero projections $e_{1}, e_{2} \in N_{2}$ with $e_{1}+e_{2}=1$. Set

$$
y:=\frac{1}{\varphi_{1}\left(e_{1}\right)} e_{1}-\frac{1}{\varphi_{1}\left(e_{2}\right)} e_{2}
$$


and then it is in $\operatorname{Ker} E \cap \tilde{N}_{2}$.

Let $x$ be an element in the center $\mathscr{Z}(\tilde{M})$, and then it is in $\tilde{N}_{1}$ by Corollary 4. We have

$$
(x-E(x)) y+E(x) y=x y=y x=y E(x)+y(x-E(x)) .
$$

By freeness with amalgamation $D$ (see (3)), the elements $(x-E(x)) y$, $y(x-E(x))$ and $\{E(x) y, y E(x)\}$ are orthogonal with respect to $E$ so that

$$
\|y(x-E(x))\|_{\psi \circ E}=0
$$

for a fixed faithful normal state $\psi$ on $\mathbf{C} \rtimes \mathbf{R}$. Thus we have

$$
\begin{aligned}
& C \times \psi \circ E\left((x-E(x))^{*} e_{i}(x-E(x))\right) \\
\leq & \psi \circ E\left((x-E(x))^{*} y^{*} y(x-E(x))\right)=0, \quad i=1,2,
\end{aligned}
$$

where $C=\min \left\{\left(\frac{1}{\varphi_{1}\left(e_{i}\right)}\right)^{2}: i=1,2\right\}$, and hence

$$
(x-E(x))^{*} e_{i}(x-E(x))=0 \quad i=1,2 .
$$

Since $e_{1}+e_{2}=1$, this implies $x-E(x)=0$, i.e., $x=E(x) \in D=\mathrm{C} \rtimes \mathrm{R}$.

The factoriality of $M$ follows from

$$
\mathscr{Z}(M)=\mathscr{Z}(\tilde{M})^{\theta^{M}} \subseteq(\mathrm{C} \rtimes \mathrm{R})^{\theta^{M}}=\mathrm{C} 1,
$$

where $\theta^{M}$ is the dual action of $M$.

In the above argument, information on the flow of weights was used to show the factoriality. However, it can be directly proved.

COROLlary 8. The above factor $M$ is not of type $\mathrm{III}_{0}$, and hence its type can be known by the Connes T-set. In this case, the Connes T-set is computed as follows:

$$
T(M)=\left\{t \in \mathrm{R}: \sigma_{t}^{\varphi}=\mathrm{Id}\right\} .
$$

Proof. By the above theorem, the (smooth) flow of weights ([CT]) of $M$ is a factor flow of the transitive one arising from $C \rtimes R$. Hence, the flow of weights of $M$ is also transitive. Thus, $M$ is not of type $\mathrm{III}_{0}$. This well-known fact can be seen as follows: Since the flow of weights is transitive, than it must be the translation on $\mathrm{R} / H$ with a closed subgroup $H$ of $\mathrm{R}$. (See $[\mathrm{M}$, $\S 2,3],[Z$, p. 373] for example.) Since any closed subgroup of $R$ is one of $\{0\}$, $(-\log \lambda) Z(0<\lambda<1)$ and $R$, the flow of weights must be a periodic flow or the trivial one, and hence $M$ is not of type $\mathrm{III}_{0}$. (This fact, i.e., $M$ is not of type $\mathrm{III}_{0}$, also follows from the fact that the flow of weights of a type $\mathrm{III}_{0}$ 
factor (i.e., an aperiodic flow) can be written as a flow built under a function with an ergodic base transformation on a non-atomic measure space. See [T4, Chap. III §3] for example.)

By symmetry we may and do assume that $\left(N_{1}\right)_{\varphi_{1}}$ contains a non-atomic von Neumann subalgebra. First we further suppose that $\left(N_{2}\right)_{\varphi_{2}} \neq \mathrm{C} 1$. Then, by the similar (actually simpler in this case) argument based on Corollary 2 as in the proof of the previous theorem we have $\left(M_{\varphi}\right)^{\prime} \cap M=\mathrm{C}$, and get the assertion on the T-set. Next, we suppose that $\left(N_{2}\right)_{\varphi_{2}}=\mathrm{C} 1$. If $\left(\left(N_{1}\right)_{\varphi_{1}}\right)^{\prime} \cap N_{1}=\mathrm{C} 1$, then the same relative commutant property as above can be obtained directly by Corollary 2 , and hence we get the assertion on the T-set. Therefore, the case that the relative commutant $\left(\left(N_{1}\right)_{\varphi_{1}}\right)^{\prime} \cap N_{1}$ is not trivial and $\left(N_{2}\right)_{\varphi_{2}}=\mathrm{C} 1$ remains. However, we can prove the following:

Claim. If $u$ is a unitary in $N_{1}$ such that $\sigma_{t}^{\varphi}=\operatorname{Ad} u$, then $u$ is in C1.

Indeed, Set $u^{\circ}:=u-\varphi_{1}(u) 1$, and let $x^{\circ}$ be a non-zero element in $N_{2}^{\circ}$. Then we compute

$$
u x^{\circ} u^{*}=\varphi_{1}(u) \varphi_{1}\left(u^{*}\right) x^{\circ}+\varphi_{1}(u) x^{\circ} u^{\circ *}+\varphi_{1}\left(u^{*}\right) u^{\circ} x^{\circ}+u^{\circ} x^{\circ} u^{\circ *} .
$$

Since the left-hand side is in $N_{1}^{\circ}$, the second, third and fourth terms in the right-hand side are zero by the structure of the free product representation. Thus we easily have $u^{\circ}=0$, i.e., $u=\varphi_{1}(u) 1$.

This claim immediately implies the assertion on the T-set.

It is unknown whether $\left(M_{\varphi}\right)^{\prime} \cap M=\mathrm{C} 1$ or not in the case that the relative commutant $\left(\left(N_{1}\right)_{\varphi_{1}}\right)^{\prime} \cap N_{1}$ is not trivial and $\left(N_{2}\right)_{\varphi_{2}}=\mathrm{C} 1$.

REMARK 9. The above result on the T-set was already obtained by $\mathrm{K}$. Dykema under a weaker condition than ours ([D1]). However, it is unknown whether the T-sets determine the types of the free products he considered there or not, and our proof gave another information on relative commutants of centralizers.

Remark 10. The problem on factoriality for free products was "almost" completed by K. Dykema ([D1], [D2]) (at least when given von Neumann algebras are factors). However, type classification for free products is obtained in only the following cases:

1. (L. Barnett, $[\mathrm{Br}]$ ) The centralizers of given faithful normal states contain "enough" unitaries;

2. (K. Dykema, [D2]) Each of given von Neumann algebras with faithful normal states is one of the following:

(i) a finite dimensional algebra with any faithful state,

(ii) $B(\mathscr{H})$ with any faithful normal state, 
(iii) a type III factor with an almost periodic state having the centralizer isomorphic to the AFD type $\mathrm{II}_{1}$ factor or to the free group factor $L\left(\mathrm{~F}_{\infty}\right)$,

(iv) a non-atomic AFD von Neumann algebra with a faithful normal tracial state,

(v) an interpolated free group factor $L\left(\mathrm{~F}_{r}\right)$ with a faithful normal tracial state,

(vi) a possibly infinite direct sum of algebras from (i)-(v).

Therefore, our Corollary 8 is a new type classification result for free products. In all the known cases the resulting free product von Neumann algebras are not of type $\mathrm{III}_{0}$ or have no type $\mathrm{III}_{0}$ direct summand. Hence it is an interesting problem to decide whether there exists a type $\mathrm{III}_{0}$ factor as a free product or not. Theorem 5.1 of [U2] might be useful for this problem. At least it suggests us that the (smooth) flows of weights of free products are factor flows of the transitive one arising from $C \rtimes R$ as in Theorem 7 except for several cases (for example, the finite dimensional case etc.).

Our Corollary 8 can be applied to many cases; for example, one of given von Neumann algebras with faithful normal states comes from a group of non-singular transformations on a Lebesgue space with a probability measure. It might be worth pointing out the following lemmas (and their corollary):

Lemma 11. Let $N$ be a $\sigma$-finite von Neumann algebra with a faithful normal state $\varphi$. If $N$ is semi-finite and has no type I direct summand, then the centralizer $N_{\varphi}$ contains a non-atomic von Neumann subalgebra.

Proof. Let $H=\frac{d \varphi}{d \operatorname{tr}}$ be the Radon-Nikodym derivative of $\varphi$ with respect to a faithful normal semi-finite trace $\operatorname{tr}$ on $N$ and $A_{0}$ be the abelian von Neumann subalgebra of $N$ generated by the spectral projections of $H$. It is known that $A_{0}$ is of the form

$$
A_{0}=A_{c} \oplus A_{d}
$$

where $A_{c}$ is non-atomic and $A_{d}$ is atomic, i.e.,

$$
A_{d}=\sum_{p \text { projection }}^{\oplus} \mathrm{C} p
$$

If $p$ is a minimal projection in $A_{d}\left(\subseteq A_{0}\right)$, then $H^{i t} p=\omega_{t} p$ for some $\omega_{t} \in \mathrm{T}$. Hence,

$$
\sigma_{t}^{\varphi}(p x p)=H^{i t} p x p H^{-i t}=\left(\omega_{t} p\right) x\left(\bar{\omega}_{t} p\right)=p x p
$$


for $x \in N$ so that $p N p$ is contained in $N_{\varphi}$. Of course, $A_{c}$ is also contained in $N_{\varphi}$. Set

$$
A=A_{c} \oplus \sum_{p}^{\oplus} p N p\left(\supseteq A_{0}\right) .
$$

Since $N$ has no type $I$ direct summand, $p N p$ is non-atomic. Therefore, $A$ is also non-atomic.

The following lemma can be easily observed based on the proof of the structure theorem of A. Connes ([C]) (and the similar argument as above):

LEMMA 12. Let $N$ be a $\sigma$-finite type III factor with a faithful normal state $\varphi$. If $N$ is not of type $\mathrm{III}_{1}$, then the centralizer $N_{\varphi}$ contains a non-atomic von Neumann subalgebra.

In the proof of the lemma the existence of a lacunary weight (or state) is crucial, and hence the above fact cannot be expected in the type $\mathrm{III}_{1}$ case. In fact, there exists a faithful normal state $\phi$ on the AFD type $\mathrm{III}_{1}$ factor $R_{\infty}$ whose centralizer is trivial ([HT]). Moreover, there exists a faithful normal state $\varphi$ on $R_{\infty}$ whose centralizer is just $M_{n}(\mathrm{C}), n \in \mathrm{N}$. Indeed, let

$$
N=R_{\infty} \otimes M_{n}(\mathrm{C}), \quad \varphi=\phi \otimes \tau,
$$

where $\tau$ is a normalized trace on $M_{n}(\mathrm{C})$. Then, $N \cong R_{\infty}$ and the centralizer $N_{\varphi}$ of $\varphi$ is the fixed-point algebra $N^{\sigma^{\varphi}}=\left(R_{\infty}\right)^{\sigma^{\phi}} \otimes M_{n}(\mathrm{C})=\mathrm{C} 1 \otimes M_{n}(\mathrm{C}) \cong$ $M_{n}(\mathrm{C})$.

By combining Theorem 7 (also Corollary 8) and the above two lemmas we have the following corollary:

COROLlaRY 13. If either $N_{1}$ or $N_{2}$ is one of the following:

(1) a $\sigma$-finite semi-finite von Neumann algebra having no type I direct summand;

(2) a $\sigma$-finite von Neumann algebra having the non-atomic center;

(3) a $\sigma$-finite type $\operatorname{III}_{\lambda}$ factor $(\lambda \neq 1)$;

(4) a possibly (infinite) direct sum of algebras from (1)-(3), then $M$ is a factor, not of type $\mathrm{III}_{0}$ and its (smooth) flow of weights can be computed as in Theorem 7 .

By the above corollary and K. Dykema's results (see Remark 10), the remaining cases of the type classification for free products of two factors are (i) both are of type $\mathrm{III}_{1}$; (ii) one is of type I and the other is of type $\mathrm{III}_{1}$. 


\section{A comment on Izumi-Longo-Popa's paper}

In their recent article [ILP], M. Izumi, R. Longo and S. Popa obtained the complete Galois correspondence for compact group minimal actions and more generally for compact Kac algebra minimal actions. The most crucial part of their arguments is to show the existence of a normal conditional expectation onto any intermediate subfactor of the irreducible inclusion arising from such an action, and hence they asked the question stated in $\S 1$. They probably conjectured that the question is not always true. However, only several partial affirmative answers (the finite Jones index case etc.) are known. In this section, we at first obtain a result on conditional expectations from free product von Neumann algebras onto their subalgebras (Theorem 14), which is of independent interest. Next, as an application of the result a counter-example to the above-mentioned question is given. The example shows that their result on the existence of conditional expectations is very non-trivial.

THEOREM 14. Let $N_{i}$ be a $\sigma$-finite von Neumann algebra with a faithful normal state $\varphi_{i}, i=1,2$, and $(M, \varphi)=\left(N_{1}, \varphi_{1}\right) *\left(N_{2}, \varphi_{2}\right)$ be the free product. Suppose that $\left(N_{1}\right)_{\varphi_{1}}$ contains a non-atomic von Neumann subalgebra and $N_{2} \neq \mathrm{C} 1$. Let $K$ be a von Neumann subalgebra of $N_{2}$, and set $L:=\left\{N_{1}, K\right\}^{\prime \prime}$. If there exists a normal conditional expectation $F: M \rightarrow L$, then

$$
\sigma_{t}^{\varphi_{2}}(K)=K, \quad t \in \mathrm{R},
$$

and $F=\mathrm{Id} * E_{K}^{N_{2}}$ in the sense of $[\mathrm{Bc}],[\mathrm{Ch}]$, where $E_{K}^{N_{2}}$ is the $\varphi_{2}$-conditional expectation from $\mathrm{N}_{2}$ onto $\mathrm{K}$.

Proof. Let $E_{1}: M \rightarrow N_{1}$ be the normal conditional expectation conditioned by $\varphi$. Since $\left(N_{1}\right)^{\prime} \cap M \subseteq N_{1}$ thanks to Corollary 2 , this is a unique normal conditional expectation thanks to Connes' result $([C])$, and hence $E_{1} \circ F=E_{1}$. Since

$$
\varphi \circ F=\left(\varphi \circ E_{1}\right) \circ F=\varphi \circ\left(E_{1} \circ F\right)=\varphi \circ E_{1}=\varphi,
$$

we conclude $\sigma_{t}^{\left.\varphi\right|_{L}}=\left.\sigma_{t}^{\varphi}\right|_{L} \quad(t \in \mathrm{R})$.

On the other hand, it is clear (by checking the freeness) that

$$
\left(L,\left.\varphi\right|_{L}\right)=\left(N_{1}, \varphi_{1}\right) *\left(K,\left.\varphi_{2}\right|_{K}\right) .
$$

Therefore, we have

$$
\begin{aligned}
\sigma_{t}^{\varphi_{2}}(x) & =\sigma_{t}^{\varphi}(x) \quad\left(x \in K \subseteq N_{2} \text { and }(1)\right) \\
& =\sigma_{t}^{\left.\varphi\right|_{L}}(x) \quad(x \in K \subseteq L) \\
& =\sigma_{t}^{\left.\varphi_{2}\right|_{K}}(x) \quad(\text { by (4) and }(1))
\end{aligned}
$$


for $x \in K$, and hence

$$
\sigma_{t}^{\varphi_{2}}(K)=\sigma_{t}^{\left.\varphi_{2}\right|_{K}}(K)=K \quad(t \in \mathrm{R}) .
$$

The formula $F=\mathrm{Id} * E_{K}^{N_{1}}$ is clear from the construction of a $\varphi$-conditional expectation in [T2].

Let $R_{\lambda}$ be the Powers factor of type $\operatorname{III}_{\lambda}(0<\lambda<1)$ with the usual product state $\phi_{\lambda}$. We define the state $\varphi_{\lambda}$ on $M_{2}(\mathrm{C})$ by

$$
\varphi_{\lambda}(\cdot)=\operatorname{tr}\left(\left[\begin{array}{cc}
\frac{1}{1+\lambda} & \\
& \frac{\lambda}{1+\lambda}
\end{array}\right] .\right) .
$$

Let

$$
(M, \varphi)=\left(R_{\lambda}, \phi_{\lambda}\right) *\left(M_{2}(\mathrm{C}), \varphi_{\lambda}\right)
$$

be the free product. It is known that $\left(R_{\lambda}\right)_{\phi_{\lambda}}$ is non-atomic (or more precisely, the AFD type $\mathrm{II}_{1}$ factor). Thus $M \supseteq N:=R_{\lambda}$ is an irreducible inclusion thanks to Corollary 2. Also, there exists a (unique faithful) normal conditional expectation $E: M \rightarrow N\left(=\operatorname{Id}_{R_{\lambda}} * \varphi_{\lambda}\right)$.

REMARK 15. By using Theorem 7 it can be proved that the factors $M \supseteq N$ have the same flow of weights, i.e.,

$$
\mathscr{Z}(\tilde{M})=\mathscr{Z}(\tilde{N}) \subseteq \mathrm{C} \rtimes \mathrm{R},
$$

where $\tilde{M}, \tilde{N}$ are as in $\S 2$. Hence $M$ and $N$ are of type III $_{\lambda}$.

$$
\text { Let } K:=\left\{\left[\begin{array}{ll}
0 & 1 \\
1 & 0
\end{array}\right]\right\}^{\prime \prime}\left(=\mathrm{C} 1+\mathrm{C}\left[\begin{array}{ll}
0 & 1 \\
1 & 0
\end{array}\right]\right) \text {, and set } L:=\{N, K\}^{\prime \prime} \text {. If there }
$$

existed a normal conditional expectation from $M$ onto $L$, then Theorem 14 would force

$$
\left[\begin{array}{ll}
\lambda^{i t} & \lambda^{-i t}
\end{array}\right]=\sigma_{t}^{\varphi_{\lambda}}\left(\left[\begin{array}{ll}
0 & 1 \\
1 & 0
\end{array}\right]\right) \in K
$$

for each $t \in \mathrm{R}$, a contradiction. Therefore, we get the following corollary:

COROllary 16. There exists no normal conditional expectation from $M$ onto $L$.

Here, the following fact might be worth pointing out:

FACT 17. Let $M \supseteq N$ be an irreducible inclusion of $\sigma$-finite factors with a (unique faithful) normal conditional expectation $E: M \rightarrow N$. If $L$ is an intermediate subfactor which is the range of a faithful normal semi-finite operator- 
valued weight from $M$, then there exists a (unique faithful) normal conditional expectation from $M$ onto $L$.

Proof. Let $F$ be a faithful normal semi-finite operator-valued weight from $M$ onto $L$. Fix a faithful normal state $\varphi$ on $N$. The restriction of $E$ to $L$ is denoted by $E_{L}$, and let $u_{t}:=\left[D \varphi \circ E: D \varphi \circ E_{L} \circ F\right]_{t}$ be the Connes cocycle Radon-Nikodym derivative. Since $\left.\sigma_{t}^{\varphi \circ E}\right|_{N}=\sigma_{t}^{\varphi}$ and $\left.\sigma_{t}^{\varphi \circ E_{L} \circ F}\right|_{N}=\sigma_{t}^{\varphi}$ (see [H]), we have

$$
\sigma_{t}^{\varphi}(x)=\sigma_{t}^{\varphi \circ E}(x)=u_{t} \sigma_{t}^{\varphi \circ E_{L} \circ F}(x) u_{t}^{*}=u_{t} \sigma_{t}^{\varphi}(x) u_{t}^{*}
$$

for $x \in N$. Thus we have $u_{t} \in N^{\prime} \cap M=\mathrm{C} 1$, and hence

$$
\sigma_{t}^{\varphi \circ E}(L)=\sigma_{t}^{\varphi \circ E_{L} \circ F}(L)=\sigma_{t}^{\varphi \circ E_{L}}(L)=L .
$$

Hence, we are done thanks to Takesaki's result ([T2]).

This fact implies that our $L$ is not the range of any faithful normal semifinite operator-valued weight from $M$. Indeed, if so was $L$, then the above fact would imply the existence of a normal conditional expectation from $M$ onto $L$. This contradicts Corollary 16.

REMARK 18. Let $M \supseteq N$ be an irreducible inclusion of $\sigma$-finite factors with a (unique faithful) normal conditional expectation and $M_{1}$ be the Jones basic extension. In [ILP], the structure of the relative commutant $N^{\prime} \cap M_{1}$ was investigated. In our case, we can show, by the same argument as in p.23 in [ILP], that the relative commutant $N^{\prime} \cap M_{1}$ has a type III $_{\lambda}$ direct summand. Indeed, let ${ }_{N} X_{N}$ be the $N-N$ bimodule generated by the element $\left[\begin{array}{ll}0 & 1 \\ 1 & 0\end{array}\right]$ of $M_{2}$ (C) regarded as a vector in $L^{2}(M, \varphi)$. Then ${ }_{N} X_{N} \cong{ }_{N} L^{2}(N) \otimes L^{2}(N)_{N}$, and hence $\operatorname{End}\left({ }_{N} X_{N}\right) \cong N^{\mathrm{op}} \otimes N$.

Remark 19. In the above example, $N$ is AFD, while $M$ is not. (See [D2].) However we can construct $M \supseteq N$ in such a way that $M$ and $N$ are isomorphic to the each other by considering the free product

$$
(N, \phi)=\underset{n=1}{\infty} \underset{n}{*}\left(M_{2}(\mathrm{C}), \varphi_{\lambda}\right),
$$

instead of the Powers factor $R_{\lambda}$ with the product state $\phi_{\lambda}$. 


\section{REFERENCES}

[Br] L. Barnett, Free product von Neumann algebras of type III, Proc. Amer. Math. Soc. 123 (1995), 543-553.

[Bc] F. Boca, Completely positive maps on amalgamated product $C^{*}$-algebras, Math. Scand. 72 (1993), 212-222.

[Ch] M. Choda, Reduced free products of completely positive maps and entropy for free product of automorphisms, Publ. Res. Inst. Math. Sci. 32 (1996), 371-382.

[C] A. Connes, Une classification des facteurs de type III, Ann. Sci. École Norm. Sup. (4) 8 (1973), 133-252.

[CT] A. Connes and M. Takesaki, The flow of weights on factors of type III, Tôhoku Math. J. 29 (1977), 473-575.

[D1] K.J. Dykema, Factoriality and Connes' invariant $T(M)$ for free products of von Neumann algebras, J. Reine Angew. Math. 450 (1994), 159-180.

[D2] K.J. Dykema, Free products of finite dimensional and other von Neumann algebras with respect to non-tracial states, Fields Inst. Comm. 12 (1996), 41-88.

[G] L. Ge, On maximal injective subalgebras of factors, Adv. Math. 118 (1996), 34-70.

[H] U. Haagerup, Operator valued weights in von Neumann algebras, I, J. Funct. Anal. 32 (1979), 175-206.

[HT] R. Herman and M. Takesaki, States and automorphism groups of operator algebras, Comm. Math. Phys. 19 (1970), 142-160.

[ILP] M. Izumi, R. Longo and S. Popa, A Galois correspondence for compact groups of automorphisms of von Neumann algebras with a generalization to Kac algebras, J. Funct. Anal. 155 (1998), 25-63.

[Kd] R. Kadison, Normalcy in operator algebras, Duke Math. J. 29 (1962), 459-464.

[KL] H. Kosaki and R. Longo, A remark on the minimal index of subfactors, J. Funct. Anal. 107 (1992), 458-470.

[M] G. Mackey, Ergodic theory and virtual groups, Math. Ann. 166 (1966), 187-207.

[P1] S. Popa, Orthogonal pairs of *-subalgebras in finite von Neumann algebras, J. Operator Theory 9 (1983), 253-268.

[P2] S. Popa, Maximal injective subalgebras in factors associated with free groups, Adv. Math. 50 (1983), 27-48.

[P3] S. Popa, Markov traces on universal Jones algebras and subfactors of finite index, Invent. Math. 111 (1993), 375-405.

[R] F. Radulescu, A type $\mathrm{III}_{\lambda}$ factor with core isomorphic to the von Neumann algebra of a free group factor tensoring $B(\mathscr{H})$, Recent advances in operator algebras (Orléans, 1992), Astérique (1995), 203-209.

[S] D. Shlyakhtenko, Free quasi-free states, Pacific J. Math. 177 (1997), 329-368.

[T1] M. Takesaki, Tomita's theory of modular Hilbert algebras and its applications, Lecture Notes in Math. 128 (1970).

[T2] M. Takesaki, Conditional expectations in von Neumann algebras, J. Funct. Anal. 9 (1972), 306-321.

[T3] M. Takesaki, Duality for crossed products and the structure of von Neumann algebras of type III, Acta Math. 131 (1973), 249-310.

[T4] M. Takesaki, Structure of Factors and Automorphism Groups, CBMS Regional Conf. Ser. in Math. 51 (1983).

[U1] Y. Ueda, A minimal action of the compact quantum group $S U_{q}(n)$ on a full factor, J. Math. Soc. Japan 51 (1999) 449-461.

[U2] Y. Ueda, Amalgamated free product over Cartan subalgebra, Pacific J. Math.191 (1999), $359-392$.

[V] D.V. Voiculescu, Symmetries of some reduced free product $C^{*}$-algebras, Operator Algebras and Their Connections with Topology and Ergodic Theory, Lecture Notes in Math. 1132, Berlin and New York, 556-588. 
REMARKS ON FREE PRODUCTS WITH RESPECT TO NON-TRACIAL STATES 125

[VDN] D.V. Voiculescu, K.J. Dykema and A. Nica, Free Random Variables, CRM Monograph Series, vol. I Amer. Math. Soc. Providence, RI, 1992.

[Z] R.Z. Zimmer, Extensions of ergodic group actions, Illinois J. Math. 20 (1976), 373-409.

GRADUATE SCHOOL OF MATHEMATICS

KYUSHU UNIVERSITY

FUKUOKA, 810-8560

JAPAN

Email: ueda@math.kyushu-u.ac.jp

CURRENT ADDRESS:

DEPARTMENT OF MATHEMATICS

GRADUATE SCHOOL OF SCIENCE

HIROSHIMA UNIVERSITY

HIGASHI-HIROSHIMA, 739-8526

JAPAN

Email: ueda@math.sci.hiroshima-u.ac.jp 